\title{
Density of bivariate homogeneous polynomials on non-convex curves*
}

\author{
András Kroó ${ }^{\dagger} \quad$ Vilmos Totik T $^{\ddagger}$
}

December 20, 2018

\begin{abstract}
The density of bivariate homogeneous polynomials is studied in the space of continuous functions on the $L_{\alpha}$ sphere given by $K_{\alpha}:=\left\{(x, y) \in \mathbb{R}^{2}:|x|^{\alpha}+|y|^{\alpha}=1\right\}, \alpha>0$. The goal is to approximate functions $f \in C\left(K_{\alpha}\right)$ by sums of the form $h_{2 n}+h_{2 n+1}$, where $h_{2 n}, h_{2 n+1}$ are bivariate homogeneous polynomials of degree $2 n$ and $2 n+1$, respectively. It is known that whenever $\alpha \geq 1$, i.e. when $K_{\alpha}$ is convex, a Weierstrass-type approximation result holds, namely for every $f \in C\left(K_{\alpha}\right)$ there are homogeneous polynomials $h_{2 n}, h_{2 n+1}$ for which $f=\lim _{n \rightarrow \infty}\left(h_{2 n}+h_{2 n+1}\right)$ uniformly on $K_{\alpha}$. In this note the problem is solved in the non-convex case $0<\alpha<1$. It is verified that $f(x, y)$ is a uniform limit on $K_{\alpha}$ of sums $h_{2 n}+h_{2 n+1}$ of homogeneous polynomials if and only if $f( \pm 1,0)=f(0, \pm 1)=0$. The theorem is proven in an equivalent form: $g \in C(\mathbb{R})$ is a uniform limit as $n \rightarrow \infty$ of weighted polynomials $\left(1+|t|^{\alpha}\right)^{-n / \alpha} p_{n}(t)$ (degree $p_{n} \leq n$ ) if and only if $g(0)=g(\infty)=g(-\infty)=0$.
\end{abstract}

\section{Introduction}

In this paper we study the approximation problem by bivariate homogeneous polynomials on certain subsets of the plane. Since homogeneous polynomials change drastically along lines passing through the origin, in general approximation can only be expected on curves symmetric onto the origin. In the present work we shall be concerned with the density of bivariate homogeneous polynomials in the space of continuous functions on the $L_{\alpha}$ sphere given by

$$
K_{\alpha}:=\left\{(x, y) \in \mathbb{R}^{2}:|x|^{\alpha}+|y|^{\alpha}=1\right\}, \quad \alpha>0 .
$$

Since, in general, both even an odd homogeneous polynomials are needed for approximation (unless the function itself is even or odd), the goal is to approximate functions $f \in C\left(K_{\alpha}\right)$ by sums of the form $h_{2 n}+h_{2 n+1}$, where $h_{2 n}, h_{2 n+1}$ are bivariate homogeneous polynomials of degree $2 n$ and $2 n+1$, respectively. It is known (see the references below) that whenever $K \subset \mathbb{R}^{2}$ is a 0 -symmetric closed convex curve, then every $f \in C(K)$ can be uniformly approximated on $K$ by sums of pairs of homogeneous polynomials $h_{n}+h_{n+1}$ as $n \rightarrow \infty$. This means that a Weierstrass-type approximation theorem holds for homogeneous polynomials on $K \subset \mathbb{R}^{2}$. In particular, when $\alpha \geq 1$, i.e. when $K_{\alpha} \subset \mathbb{R}^{2}$ is convex, every $f \in C\left(K_{\alpha}\right)$ is a uniform limit of sums of homogeneous polynomials $h_{n}+h_{n+1}$ as $n \rightarrow \infty$. The main goal of this paper is to solve the non-convex case $0<\alpha<1$. It will turn out that in this case some additional restrictions are needed to be imposed on the function in order for a Weierstrass-type approximation theorem to hold. Namely, we will verify that only functions vanishing at the non-smooth points ("corners") of the boundary can be properly approximated.

The just mentioned Weierstrass-type result for convex curves was verified independently by Benko and Kroó [1] and Varjú [9] using methods of potential theory. Subsequently Totik [8] provided a different proof which was not based on potential theoretical methods. The corresponding general conjecture for 0-symmetric convex surfaces in $\mathbb{R}^{d}, d>2$ (resolved for boundaries of polytopes [9] and for regular convex surfaces [4]) is still open in its full generality, see [4] and [8] for details.

It is well known (see e.g. [9]) that the above-formulated approximation problem on $K_{\alpha}$ is equivalent to approximating even functions by even homogeneous polynomials $h_{2 n}(x, y)=\sum_{k=0}^{2 n} a_{k} x^{2 n-k} y^{k}$. In turn, making the

\footnotetext{
*AMS Subject classification: 41A10, 41A63. Key words and phrases: bivariate homogeneous polynomials, approximation by weighted polynomials, $L_{\alpha}$ spheres

${ }^{\dagger}$ Supported by the NKFIH - OTKA Grant K111742

‡Supported by NSF grant DMS 1564541
} 
substitution $y=t x, x \geq 0, t \in \mathbb{R}$, one can easily see that this is equivalent to approximating real continuous functions $f(t)$ on $\mathbb{R}$ which have equal finite limits at $\infty$ and $-\infty$ by weighted polynomials $w_{\alpha}(t)^{2 n} p_{2 n}(t), p_{2 n} \in P_{2 n}, n \in \mathbb{N}$, where

$$
w_{\alpha}(t):=\left(1+|t|^{\alpha}\right)^{-1 / \alpha},
$$

and $P_{n}$ denotes the set of univariate real algebraic polynomials of degree at most $n$. Note that here the weight $w_{\alpha}^{2 n}$ changes with the degree $2 n$ of the polynomial $p_{2 n}$, and actually slight variations in $w_{\alpha}$ are enlarged by the $2 n$-th power. It is known that for certain weight functions this so-called weighted polynomial approximation with varying weights is closely related to logarithmic potentials with external fields, see Sections VI.1-2 in the book [7]. However, the present weight function $w_{\alpha}$ is not admissible in the sense of [7], so the classical theory is not directly applicable in the present case.

Let us denote by $C(\mathbb{R})$ the space of real continuous functions $f(x)$ on $\mathbb{R}$ which have equal finite limits at $\infty$ and $-\infty$ (denoted by $f(\infty)$ and $f(-\infty)$, respectively), i.e., $f(\infty)=f(-\infty)$. In addition, we set

$$
C_{0}(\mathbb{R}):=\{f \in C(\mathbb{R}): f(0)=f(\infty)=f(-\infty)=0\} .
$$

The main result of this paper is the following.

Theorem 1 Let $0<\alpha<1$. Then there exist weighted polynomials $w_{\alpha}^{n} p_{n}, p_{n} \in P_{n}, n \in \mathbb{N}$, converging to $f(x)$ uniformly on $\mathbb{R}$ if and only if $f \in C_{0}(\mathbb{R})$.

The statement is also true if $n \in \mathbb{N}$ is replaced in it by $n \in 2 \mathbb{N}$ (the necessity will be proven in that form), and it as indicated above this has then an immediate implication for homogeneous approximation on $K_{\alpha}$.

Corollary 2 Let $0<\alpha<1$. In order that $f(x, y) \in C\left(K_{\alpha}\right)$ be a uniform limit on $K_{\alpha}$ of sums of homogeneous polynomials $h_{n}+h_{n+1}$ of degree $n$ and $n+1$, it is necessary and sufficient that $f( \pm 1,0)=f(0, \pm 1)=0$.

It should be noted that the necessity part in the above corollary immediately extends to the case of $d>2$ variables since the restriction of a homogeneous polynomial to any 2-dimensional coordinate plane is clearly a bivariate homogeneous polynomial of the same degree.

The paper is organized as follows. First we verify the necessity part in Theorem 1 which will be based on a Markov-type result for weighted polynomials (see Lemma 3 below). Then we will proceed by proving the sufficiency using the theory of logarithmic potential theory with external fields. Finally, in the last section we shall present a concrete construction that proves the sufficiency - without the use of potential theory - in the case when $0<\alpha<1$ is rational.

\section{Proof of Theorem 1}

\section{Necessity}

The proof of the necessity is based on the next lemma providing a Markov-type estimate for the derivatives of weighted polynomials $w_{\alpha}^{n} p_{n}, 0<\alpha<1$, which is of independent interest. We also mention that the estimate is sharp, see the very end of the paper.

Lemma 3 Let $0<\alpha<1$, and assume that $p_{n} \in P_{n}, n \in \mathbb{N}$, satisfy

$$
w_{\alpha}^{n}(x)\left|p_{n}(x)\right| \leq M, \quad x \in \mathbb{R} .
$$

Then

$$
w_{\alpha}^{n}(x)\left|p_{n}^{\prime}(x)\right| \leq c_{\alpha} M n^{\frac{1}{\alpha}}, \quad x \in \mathbb{R} .
$$

Proof. First we verify that (2) holds at the origin. Without the loss of generality we may assume that $M=1$. By [2, p. 92.] we have with $z=u+i v$

$$
\log \left|p_{n}(z)\right| \leq \frac{|v|}{\pi} \int_{\mathbb{R}} \frac{\log \left|p_{n}(t)\right|}{(t-u)^{2}+v^{2}} d t \leq \frac{|v| n}{\pi \alpha} \int_{\mathbb{R}} \frac{\log \left(1+|t|^{\alpha}\right)}{(t-u)^{2}+v^{2}} d t=
$$




$$
\frac{n}{\pi \alpha} \int_{\mathbb{R}} \frac{\log \left(1+|u+v t|^{\alpha}\right)}{t^{2}+1} d t \leq \frac{n|z|^{\alpha}}{\pi \alpha} \int_{\mathbb{R}} \frac{1+|t|^{\alpha}}{t^{2}+1} d t=c_{\alpha} n|z|^{\alpha},
$$

where we used that, because of $0<\alpha<1$,

$$
\log \left(1+|u+v t|^{\alpha}\right) \leq|u+v t|^{\alpha} \leq|u|^{\alpha}+|v|^{\alpha}|t|^{\alpha} \leq|z|^{\alpha}\left(1+|t|^{\alpha}\right) .
$$

Hence, $\left|p_{n}(z)\right| \leq e^{c_{\alpha}}$ in the disc $|z| \leq n^{-\frac{1}{\alpha}}$. Therefore, by the Cauchy integral formula,

$$
\left|p_{n}^{\prime}(0)\right| \leq e^{c_{\alpha}} n^{\frac{1}{\alpha}}
$$

which is the required estimate for $x=0$.

Now let $x=a \in \mathbb{R}$. For the given $p_{n} \in P_{n}$ satisfying (1) set $g_{n}(x):=p_{n}(x+a)$. Then

$$
\frac{\left|g_{n}(x)\right|}{\left(1+|x|^{\alpha}\right)^{n / \alpha}} \leq \frac{\left|p_{n}(x+a)\right|}{\left(1+|x+a|^{\alpha}\right)^{n / \alpha}}\left(\frac{1+|x+a|^{\alpha}}{1+|x|^{\alpha}}\right)^{n / \alpha} \leq\left(\frac{1+|x+a|^{\alpha}}{1+|x|^{\alpha}}\right)^{n / \alpha} \leq\left(1+|a|^{\alpha}\right)^{n / \alpha} .
$$

Using now the just established estimate for the derivative at $x=0$ for $g_{n}$ with $M:=\left(1+|a|^{\alpha}\right)^{n / \alpha}$, we obtain

$$
\left|p_{n}^{\prime}(a)\right|=\left|g_{n}^{\prime}(0)\right| \leq e^{c_{\alpha}} n^{\frac{1}{\alpha}}\left(1+|a|^{\alpha}\right)^{n / \alpha}, \quad a \in \mathbb{R},
$$

which completes the proof.

Iterating Lemma 3 easily yields a Markov-type result for higher order derivatives.

Corollary 4 Under the conditions of Lemma 3 we have

$$
w_{\alpha}^{n}(x)\left|p_{n}^{(k)}(x)\right| \leq c_{\alpha}^{k} M n^{\frac{k}{\alpha}}, \quad x \in \mathbb{R}, k \in \mathbb{N} .
$$

In particular,

$$
\left|p_{n}^{(k)}(0)\right| \leq c_{\alpha}^{k} M n^{\frac{k}{\alpha}}, \quad k \in \mathbb{N} .
$$

Now the necessity in Theorem 1 is an immediate consequence of the next proposition.

Proposition 5 Assume that $0<\alpha<1$ and weighted polynomials $w_{\alpha}^{2 n} p_{2 n}, p_{2 n} \in P_{2 n}, n \in \mathbb{N}$, converge to $f$ uniformly on $\mathbb{R}$. Then

$$
f(0)=\lim _{x \rightarrow \infty} f(x)=\lim _{x \rightarrow-\infty} f(x)=0 .
$$

Proof. Obviously we must have $f \in C(\mathbb{R})$. Assume first that $f(0)=a \neq 0$. Set

$$
f_{n}(x):=f\left(n^{-\frac{1}{\alpha}} x\right), \quad g_{n}(x):=p_{2 n}\left(n^{-\frac{1}{\alpha}} x\right) \in P_{2 n}, \quad n \in \mathbb{N} .
$$

By the continuity of $f$ we have

$$
\epsilon_{n}:=\max _{|x| \leq 1}\left|f_{n}(x)-a\right|=\max _{|x| \leq 1}\left|f\left(n^{-\frac{1}{\alpha}} x\right)-f(0)\right| \rightarrow 0, \quad n \rightarrow \infty .
$$

Furthermore, the convergent sequence of weighted polynomials $w_{\alpha}^{2 n} p_{2 n}$ is uniformly bounded on the real line, i.e., with some $M>0$ we have

$$
w_{\alpha}^{2 n}(x)\left|p_{2 n}(x)\right| \leq M, \quad x \in \mathbb{R}, n \in \mathbb{N} .
$$

Hence, by Corollary 4,

$$
\left|g_{n}^{(k)}(0)\right|=\left|n^{-\frac{k}{\alpha}} p_{2 n}^{(k)}(0)\right| \leq M^{k} c_{\alpha}^{k}, \quad k, n \in \mathbb{N} .
$$

Note that the right hand side is independent of $n$. 
Setting $g_{n}(x):=\sum_{k=0}^{n} b_{k, n} x^{k}$ it follows that

$$
\left|b_{k, n}\right|=\frac{\left|g_{n}^{(k)}(0)\right|}{k !} \leq \frac{M^{k} c_{\alpha}^{k}}{k !} \leq\left(\frac{4 M c_{\alpha}}{k}\right)^{k}
$$

Therefore, with any fixed integer $m>8 M c_{\alpha}$, we have that whenever $|x| \leq 1$ and $n \in \mathbb{N}$

$$
g_{n}(x):=\sum_{k=0}^{n} b_{k, n} x^{k}=\sum_{k=0}^{m} b_{k, n} x^{k}+\sum_{k=m+1}^{n} b_{k, n} x^{k}=g_{m, n}(x)+O\left(2^{-m}\right),
$$

where $g_{m, n} \in P_{m},\left|g_{m, n}(x)\right| \leq c_{\alpha, M}$.

Set

$$
\delta_{n}:=\max _{x \in \mathbb{R}}\left|\frac{p_{2 n}(x)}{\left(1+|x|^{\alpha}\right)^{2 n / \alpha}}-f(x)\right| \rightarrow 0, \quad n \rightarrow \infty .
$$

Then the above estimate together with (3) and (4) yields

$$
\begin{gathered}
\delta_{n} \geq \max _{x \in \mathbb{R}}\left|\frac{p_{2 n}\left(n^{-\frac{1}{\alpha}} x\right)}{\left(1+\frac{|x|^{\alpha}}{n}\right)^{2 n / \alpha}}-f\left(n^{-\frac{1}{\alpha}} x\right)\right| \geq \max _{|x| \leq 1}\left|\frac{g_{n}(x)}{\left(1+\frac{|x|^{\alpha}}{n}\right)^{2 n / \alpha}}-f_{n}(x)\right| \\
\geq \max _{|x| \leq 1}\left|\frac{g_{m, n}(x)}{\left(1+\frac{|x|^{\alpha}}{n}\right)^{2 n / \alpha}}-a\right|-\epsilon_{n}-c 2^{-m} .
\end{gathered}
$$

Now letting $n \rightarrow \infty$ in the last estimate and using that $g_{m, n} \in P_{m},\left|g_{m, n}(x)\right| \leq c_{\alpha, M}, n \in \mathbb{N}$, hence $\left\{g_{m, n}\right\}_{n=1}^{\infty}$ contains a locally convergent subsequence, we obtain that for some $g_{m} \in P_{m}$

$$
\max _{|x| \leq 1}\left|g_{m}(x)-a e^{2|x|^{\alpha} / \alpha}\right|=O\left(2^{-m}\right), \quad m>8 M c_{\alpha}
$$

Now we need to recall that, in view of a classical result of Bernstein (see e.g. [3, Theorem 7.8.1]), such an exponential rate of approximation is possible only for analytic functions but not for $e^{2|x|^{\alpha} / \alpha}$. Hence, we must have $a=0$, i.e. $f(0)=0$.

Furthermore, since $f$ is a uniform limit of weighted polynomials $w_{\alpha}^{2 n} p_{2 n}$ and each of them has equal limits at $\infty$ and $-\infty$ it follows that $f$ has a limit at $\pm \infty$, and $\lim _{x \rightarrow \infty} f(x)=\lim _{x \rightarrow-\infty} f(x)$. Then the function $g(x):=f(1 / x)$ is also continuous on the real line, and it is the uniform limit of the weighted polynomials $w_{\alpha}^{2 n}(x) x^{2 n} p_{2 n}(1 / x):=$ $w_{\alpha}^{2 n}(x) p_{2 n}^{*}(x), p_{2 n}^{*} \in P_{2 n}$. Hence, by what we have proven above, we must have $g(0)=0$, i.e.,

$$
\lim _{x \rightarrow \infty} f(x)=\lim _{x \rightarrow-\infty} f(x)=0=f(0) .
$$

\section{Sufficiency}

In the proof of the sufficiency we shall need the basics of logarithmic potential theory, see e.g. the books [6] and [7] for them.

For a $0<\gamma<\alpha / 2$ consider the weight $W_{0}(x)=1 /\left(1+x^{\alpha / 2}\right)^{1 / \gamma}$ on $[0, \infty)$. This $W_{0}$ is admissible in the sense of [7]. Let $S_{W_{0}}$ be the compact support of the associated equilibrium measure $\mu_{W_{0}}$ (see [7, Ch. I]). Since $W_{0}(0)>W_{0}(x)$ for all $x>0$, we have $0 \in S_{W_{0}}\left(\left[7\right.\right.$, Theorem IV.1.3]). Setting $W_{0}(x)=e^{-Q_{0}(x)}$, with $Q_{0}(x):=(1 / \gamma) \log \left(1+x^{\alpha / 2}\right)$ we have that

$$
x Q_{0}^{\prime}(x)=\frac{\alpha}{2 \gamma} \frac{x^{\alpha / 2}}{1+x^{\alpha / 2}}
$$

is increasing in $(0, \infty)$. Therefore, by $\left[7\right.$, Theorem IV.1.10(c)], the support $S_{W_{0}}$ is a certain interval $\left[0, b_{\gamma}\right]$, where the endpoint $b_{\gamma}:=b$ satisfies the equation

$$
\frac{1}{\pi} \int_{0}^{b} \frac{\alpha}{2 \gamma} \frac{x^{\alpha / 2-1}}{1+x^{\alpha / 2}} \sqrt{\frac{x}{b-x}} d x=1
$$


see [7, Theorem IV.1.11(i)]. Using the substitution $x=b t$ the above equation can be written in the form

$$
\frac{1}{\pi} \int_{0}^{1} \frac{b^{\alpha / 2} t^{\alpha / 2}}{1+b^{\alpha / 2} t^{\alpha / 2}} \frac{1}{\sqrt{t(1-t)}} d t=\frac{2 \gamma}{\alpha}
$$

Since

$$
\frac{1}{\pi} \int_{0}^{1} \frac{1}{\sqrt{t(1-t)}} d t=1
$$

and the integrand in (5) is strictly less than $1 / \sqrt{t(1-t)}$ and monotonically tends to that function as $b \rightarrow \infty$, it follows from the monotone convergence theorem that $b_{\gamma} \rightarrow \infty$ as $\gamma \rightarrow \alpha / 2$. It also follows that $b_{\gamma}$ is an increasing and continuous function of $\gamma$.

If $\mu_{W_{0}}$ is the equilibrium measure, then it is locally absolutely continuous on $S_{W_{0}}$ and its density $v_{0}$ is continuous on $\left(0, b_{\gamma}\right)$, i.e. on the (one dimensional) interior of $S_{W_{0}}$ (see [7, Theorem IV.2.5]). Furthermore, $v_{0}$ is strictly positive on $\left(0, b_{\gamma}\right)$. Indeed, let $\lambda>1$ and consider the weight $W_{0}(x)^{\lambda}=1 /\left(1+x^{\alpha / 2}\right)^{\lambda / \gamma}$. This $W_{0}^{\lambda}$ is a weight similar to $W_{0}$ but with the parameter $\gamma / \lambda$ replacing $\gamma$. Therefore the above observations extend to the weight $W_{0}^{\lambda}$, as well. Thus it follows from what we have done above that $S_{W_{0}^{\lambda}}=\left[0, b_{\gamma / \lambda}\right] \subset\left[0, b_{\gamma}\right]=S_{W_{0}}$. In view of $[7$, Theorem IV.4.9] we have on $S_{W_{0}^{\lambda}}$ the inequality

$$
\mu_{W_{0}} \geq \frac{1}{\lambda} \mu_{W_{0}^{\lambda}}+\left(1-\frac{1}{\lambda}\right) \omega_{S_{W_{0}}}
$$

where $\omega_{I}$ denotes the equilibrium measure of the set $I$. Since (see e.g. [7, (I.1.7)])

$$
d \omega_{S_{W_{0}}}(t)=d \omega_{\left[0, b_{\gamma}\right]}(t)=\frac{1}{\pi \sqrt{t\left(b_{\gamma}-t\right)}} d t
$$

the positivity of $v_{0}$ on the interior of $S_{W_{0}^{\lambda}}$, i.e. on $\left(0, b_{\gamma / \lambda}\right)$ follows. But here $b_{\gamma / \lambda} \rightarrow b_{\gamma}$ if $\lambda \searrow 1$, so the positivity of $v_{0}$ on the whole interval $\left(0, b_{\gamma}\right)$ follows, as well.

Consider now for a $0<\beta \leq \alpha$ the weight functions $W_{\beta}(x)=1 /\left(1+|x|^{\alpha}\right)^{1 / \beta}$ on $\mathbb{R}$. When $\beta=\alpha$ we have $W_{\alpha}=w_{\alpha}$, which is the weight function in Theorem 1 . This is no longer admissible in the sense of [7] since $|x| w_{\alpha}(x)$ does not tend to 0 as $|x| \rightarrow \infty$. Nevertheless, the claim in the theorem is that even with this weight every $f \in C_{0}(\mathbb{R})$ is the uniform limit on $\mathbb{R}$ of weighted polynomials $p_{n} w_{\alpha}^{n}, p_{n} \in P_{n}$ as $n \rightarrow \infty$.

To prove that note that for $\beta<\alpha$ the weight function $W_{\beta}$ is admissible, and symmetric with respect to the origin, hence if we set $\gamma=\beta / 2$ above, then, in view of [7, Theorem IV.1.10(f)], $S_{W_{\beta}}=\left[-\sqrt{b_{\beta / 2}}, \sqrt{b_{\beta / 2}}\right]$ and $d \mu_{W_{\beta}}(t)=d \mu_{W_{0}}\left(t^{2}\right) / 2$. Thus, if $v_{\beta}$ is the density of $\mu_{W_{\beta}}$, then $v_{\beta}$ is continuous and strictly positive on $D_{\beta}:=$ $\left(-\sqrt{b_{\beta / 2}}, 0\right) \cup\left(0, \sqrt{b_{\beta / 2}}\right)$. But then we get from [7, Theorem VI.1.5] that every function $g \in C(\mathbb{R})$ which vanishes outside $D_{\beta}$ is the uniform limit of weighted polynomials $W_{\beta}^{m} p_{m}, p_{m} \in P_{m}, m=1,2, \ldots$. If we apply this to $m=$ $[n(\beta / \alpha)]$ for a given $n=1,2, \ldots$, then it follows that for $n=1,2, \ldots$ there are weighted polynomials $W_{\beta}^{[n(\beta / \alpha)]} p_{[n(\beta / \alpha)]}$ that converge to $g$ uniformly on $\mathbb{R}$. Here $p_{[n(\beta / \alpha)]}$ is of degree $\leq n$ and

$$
W_{\beta}(x)^{[n(\beta / \alpha)]}=\frac{1}{\left(1+|x|^{\alpha}\right)^{[n(\beta / \alpha)] / \beta}}=W_{\alpha}(x)^{n} \frac{1}{\left(1+|x|^{\alpha}\right)^{\tau_{n}}}
$$

where

$$
\tau_{n}:=\frac{1}{\beta}([n \beta / \alpha]-n \beta / \alpha), \quad-1 / \beta \leq \tau_{n} \leq 0 .
$$

If we choose here $\beta<\alpha$ so that $\frac{\beta}{\alpha}=\frac{p}{q}$ is rational, then using $(7)$ it follows that for any $n \equiv s(\bmod q)$, i.e. for any $n=k q+s, k \in \mathbb{N}, 0 \leq s \leq q-1$, we have

$$
\tau_{n}=\frac{1}{\beta}([n \beta / \alpha]-n \beta / \alpha)=\frac{1}{\beta}([s \beta / \alpha]-s \beta / \alpha):=\tau_{s},
$$

i.e., $\tau_{n}=\tau_{s}$ is independent of $n$ whenever $n=k q+s$ with any $k \in \mathbb{N}$ and a fixed $0 \leq s \leq q-1$.

Hence, by (6), for every function $g_{0} \in C(\mathbb{R})$ which vanishes outside $D_{\beta}$ and every $n \equiv s(\bmod q)$, there exist polynomials $p_{n}$ of degree at most $n$ such that $W_{\alpha}(x)^{n} p_{n}(x)\left(1+|x|^{\alpha}\right)^{-\tau_{s}} \rightarrow g_{0}$ uniformly on $\mathbb{R}$. Since $\tau_{s} \leq 0$ and $W_{\alpha}=w_{\alpha}$, this implies that $w_{\alpha}(x)^{n} p_{n}(x) \rightarrow\left(1+|x|^{\alpha}\right)^{\tau_{s}} g_{0}(x)$ uniformly on $\mathbb{R}$ for any $g_{0}$ which is zero outside $D_{\beta}$. 
Setting now $g:=g_{0}\left(1+|x|^{\alpha}\right)^{\tau_{s}}$ we get the required approximation statement for every function $g \in C(\mathbb{R})$ which vanishes outside $D_{\beta}$ and for every $n \equiv s(\bmod q)$ with any fixed $0 \leq s \leq q-1$ (i.e. for all such $n$ there are weighted polynomials $w_{\alpha}^{n} p_{n}$ that converge uniformly on $\mathbb{R}$ to $g$ ). Evidently, this yields the required statement for every $n \in \mathbb{N}$, as well, i.e., we are done in the case when $g \in C(\mathbb{R})$ vanishes outside $D_{\beta}$.

Finally, it remains to note that $b_{\beta / 2}$ can be made as large as we wish by letting $\beta \nearrow \alpha$ (besides the requirement that $\frac{\beta}{\alpha}$ is a rational number). In addition, every $f \in C_{0}(\mathbb{R})$ is the uniform limit on $\mathbb{R}$ of functions $g \in C(\mathbb{R})$ which vanish outside some $D_{\beta}, \beta<\alpha$. Now a standard diagonalization process yields that every $f \in C_{0}(\mathbb{R})$ is the uniform limit on $\mathbb{R}$ of weighted polynomials $p_{n} w_{\alpha}^{n}$.

\section{A concrete construction}

Approximating by weighted polynomials $w^{n} p_{n}$ with varying weights is a rather non-trivial subject, and it is quite rare that in that theory concrete approximating polynomials can be given. Therefore, it is instructive to give such concrete polynomials in the present case at least when $\alpha$ is rational. In this section we present this explicit construction.

Lemma 6 Let $\alpha=\frac{p}{q}, p, q \in \mathbb{N}$ and $t_{k}:=e^{\frac{\pi k i}{q}}, 1 \leq k \leq 2 q$, be the $2 q$-th roots of unity. Then for any $n \in \mathbb{N}$

$$
g_{p n}(x):=|x|^{\alpha} \sum_{k=1}^{2 q} t_{k}\left(1+t_{k}|x|^{\alpha}\right)^{q n} \in P_{p n}
$$

Proof. We use the relation

$$
\sum_{k=1}^{2 q} t_{k}^{l}=0
$$

which holds for any integer $l \neq 2 r q, r \in \mathbb{N}$. To verify that, it suffices to note that, by the periodicity of roots of unity, $\sum_{k=1}^{2 q} t_{k}^{l}=t_{1}^{l} \sum_{k=1}^{2 q} t_{k}^{l}$. Hence if $t_{1}^{l} \neq 1$, then the above relation must hold. In addition, we evidently have $\sum_{k=1}^{2 q} t_{k}^{l}=2 q$ whenever $l=2 r q, r \in \mathbb{N}$.

Using these relations and the binomial formula we have for any $y \in \mathbb{R}$

$$
\sum_{k=1}^{2 q} t_{k}\left(1+t_{k} y\right)^{n q}=\sum_{k=1}^{2 q} \sum_{j=0}^{n q}\left(\begin{array}{c}
n q \\
j
\end{array}\right) t_{k}^{j+1} y^{j}=\sum_{j=0}^{n q}\left(\begin{array}{c}
n q \\
j
\end{array}\right) y^{j} \sum_{k=1}^{2 q} t_{k}^{j+1}=\sum_{2 r \leq n} a_{r, n} y^{2 r q-1}
$$

where $a_{r, n}:=2 q\left(\begin{array}{c}n q \\ 2 r q-1\end{array}\right)$.

Hence setting $y:=|x|^{\alpha}=|x|^{\frac{p}{q}}$ we obtain

$$
|x|^{\alpha} \sum_{k=1}^{2 q} t_{k}\left(1+t_{k}|x|^{\alpha}\right)^{q n}=|x|^{p / q} \sum_{2 r \leq n} a_{r, n}|x|^{\frac{p}{q}(2 r q-1)}=\sum_{2 r \leq n} a_{r, n} x^{2 r p} \in P_{p n},
$$

which completes the proof of the lemma.

Lemma 7 Let $\alpha=\frac{p}{q}, p, q \in \mathbb{N}$ and $g_{p n} \in P_{p n}$ be the polynomial given by (8). Then with any $s \geq 2 \alpha$ we have

$$
\left|\frac{g_{p n}(x)}{\left(1+|x|^{\alpha}\right)^{(p n+s) / \alpha}}-\frac{|x|^{\alpha}}{\left(1+|x|^{\alpha}\right)^{s / \alpha}}\right| \leq \frac{8 q}{n}, \quad x \in \mathbb{R}, n \in \mathbb{N} .
$$

Proof. We will apply below the following identity which holds for any given $a>0$ and $t_{k}=e^{\frac{\pi k i}{q}}$ :

$$
\left|1+t_{k} a\right|^{2}=\left(1+a \Re t_{k}\right)^{2}+a^{2}\left(\Im t_{k}\right)^{2}=(1+a)^{2}+2 a \Re t_{k}-2 a=(1+a)^{2}-4 a \sin ^{2} \frac{\pi k}{2 q}, 1 \leq k \leq 2 q
$$


We have by (8)

$$
\frac{g_{p n}(x)}{\left(1+|x|^{\alpha}\right)^{(p n+s) / \alpha}}=\frac{|x|^{\alpha}}{\left(1+|x|^{\alpha}\right)^{s / \alpha}}+\frac{|x|^{\alpha}}{\left(1+|x|^{\alpha}\right)^{s / \alpha}} \sum_{k=1}^{2 q-1} t_{k}\left(\frac{1+t_{k}|x|^{\alpha}}{1+|x|^{\alpha}}\right)^{q n}=\frac{|x|^{\alpha}}{\left(1+|x|^{\alpha}\right)^{s / \alpha}}+R .
$$

Now in order to verify the claim of the lemma we estimate the remainder term $R$ using (11) with $a:=|x|^{\alpha}$ :

$$
\begin{gathered}
|R| \leq \frac{|x|^{\alpha}}{\left(1+|x|^{\alpha}\right)^{s / \alpha}} \sum_{k=1}^{2 q-1}\left|\frac{1+t_{k}|x|^{\alpha}}{1+|x|^{\alpha}}\right|^{q n} \leq \frac{|x|^{\alpha}}{\left(1+|x|^{\alpha}\right)^{2}} \sum_{k=1}^{2 q-1}\left(1-\frac{4|x|^{\alpha}}{\left(1+|x|^{\alpha}\right)^{2}} \sin ^{2} \frac{\pi k}{2 q}\right)^{q n / 2} \\
\leq u \sum_{k=1}^{2 q-1}\left(1-u \sin ^{2} \frac{\pi k}{2 q}\right)^{q n / 2}, \quad u:=\frac{4|x|^{\alpha}}{\left(1+|x|^{\alpha}\right)^{2}} .
\end{gathered}
$$

Here $0 \leq u \leq 1$, and $u(1-c u)^{q n / 2} \leq \frac{2}{c q n}$ for any such $u$ and $0<c \leq 1$. Using this upper bound with $c:=\sin ^{2} \frac{\pi k}{2 q}$ we obtain

$$
|R| \leq \frac{2}{q n} \sum_{k=1}^{2 q-1} \sin ^{-2} \frac{\pi k}{2 q} \leq \frac{4}{q n} \sum_{k=1}^{q} \sin ^{-2} \frac{\pi k}{2 q} .
$$

Now we can use that $\sin \frac{\pi k}{2 q} \geq \frac{k}{q}, 1 \leq k \leq q$, which implies

$$
|R| \leq \frac{4}{q n} \sum_{k=1}^{q} \frac{q^{2}}{k^{2}}=\frac{4 q}{n} \sum_{k=1}^{q} \frac{1}{k^{2}} \leq \frac{8 q}{n} .
$$

As an easy corollary of Lemma 7 we get both even and odd test functions which can be approximated by weighted polynomials.

Corollary 8 Let $\alpha=\frac{p}{q}, p, q \in \mathbb{N}$ and $g_{p n} \in P_{p n}$ be the polynomial given by (8). Then with any $m \geq 0$ and $s \geq 2 \alpha+m$ we have

$$
\left|\frac{x^{m} g_{p n}(x)}{\left(1+|x|^{\alpha}\right)^{(p n+s) / \alpha}}-\frac{x^{m}|x|^{\alpha}}{\left(1+|x|^{\alpha}\right)^{s / \alpha}}\right| \leq \frac{8 q}{n}, \quad x \in \mathbb{R}, n \in \mathbb{N} .
$$

Now the proof of the sufficiency in Theorem 1 can be finalized by using a Stone-Weierstrass-type argument. For any closed subalgebra of functions $A \subset C(K)$ denote by $Z_{A}:=\{x \in K: g(x)=0$, for all $g \in A\}$ the zero set of $A$. Then the Stone-Weierstrass theorem (see e.g., [3, p. 13]) states that if $A$ separates points in $K \backslash Z_{A}$, then any $f \in C(K)$ which vanishes on $Z_{A}$ belongs to $A$, i.e., $A=\left\{f \in C(K): f=0\right.$ on $\left.Z_{A}\right\}$. The idea of applying the Stone-Weierstrass theorem in weighted polynomial approximation goes back to Kuijlaars [5]. We will adopt it in the proof of the next proposition, which provides the promised sufficiency part in Theorem 1 in case of rational $0<\alpha<1$.

Proposition 9 Let $\alpha$ be rational. Then given any $f \in C_{0}(\mathbb{R})$, there exist polynomials $g_{n} \in P_{n}, n=1,2, \ldots$, such that $w_{\alpha}^{n} g_{n} \rightarrow f, n \rightarrow \infty$ uniformly on $\mathbb{R}$.

Proof. Let $\alpha=\frac{p}{q}, p, q \in \mathbb{N}, 0<\alpha<1$. The proof will be accomplished in two steps. First we will verify the statement of the proposition for the subsequence $\mathbb{N}_{p}:=\{p n: n \in \mathbb{N}\}$.

Set

$$
A_{p}:=\left\{f \in C(\mathbb{R}): f=\lim _{n \rightarrow \infty} w_{\alpha}^{p n} g_{p n}, \quad g_{p n} \in P_{p n}\right\} .
$$

Obviously, $A_{p}$ is a closed subalgebra of $C(\mathbb{R})$, where, by Proposition 5 , we have $0, \infty \in Z_{A_{p}}$. Moreover, using (12) with any $s:=p r>m+2, r \in \mathbb{N}$ it follows that

$$
f_{m, s}(x):=x^{m}|x|^{\alpha} w_{\alpha}(x)^{s} \in A_{p}, \quad m \geq 0 .
$$


Since these test functions vanish only at 0 and $\infty$, we have $Z_{A_{p}}=\{0, \infty\}$. Now it remains to show that elements of $A_{p}$ separate points in $\mathbb{R} \backslash\{0, \infty\}$. Obviously, for any distinct points $x, y>0$ either $f_{0, p}(x) \neq f_{0, p}(y)$ or $f_{0,2 p}(x) \neq f_{0,2 p}(y)$, where $f_{0, p}, f_{0,2 p} \in A_{p}$ are even test functions. Clearly, the same holds for the odd test functions $f_{1, p}, f_{1,2 p} \in A_{p}$. Thus elements of $A_{p}$ separate points in $\mathbb{R} \backslash\{0, \infty\}$. Hence, by the Stone-Weierstrass theorem, we obtain that any continuous function on $\mathbb{R}$ which vanishes at $0, \infty$ is in $A_{p}$, i.e., $A_{p}=C_{0}(\mathbb{R})$. Therefore, for any $0 \leq j \leq p-1, k \geq 0$ and $s>p+k$ there exist $g_{j, n} \in P_{p n}$ such that

$$
w_{\alpha}(x)^{p n} g_{j, n}(x) \rightarrow x^{k}|x|^{\alpha} w_{\alpha}(x)^{s-j} \in C_{0}(\mathbb{R}), \quad n \rightarrow \infty .
$$

Of course, this implies

$$
w_{\alpha}(x)^{p n+j} g_{j, n}(x) \rightarrow x^{k}|x|^{\alpha} w_{\alpha}(x)^{s} \in C_{0}(\mathbb{R}), \quad 0 \leq j \leq p-1, n \rightarrow \infty .
$$

Now for an $m \in \mathbb{N}, m \equiv j(\bmod p)$, say for $m=p n+j$ with some $n \in \mathbb{N}$ and $0 \leq j \leq p-1$, set

$$
g_{m}:=g_{j, n} \in P_{p n} \subset P_{m} .
$$

Then the last relation means that for any $k \geq 0$ and $s>p+k$ we found polynomials $g_{m} \in P_{m}, m=1,2, \ldots$, such that

Therefore, if we define

$$
w_{\alpha}(x)^{m} g_{m}(x) \rightarrow x^{k}|x|^{\alpha} w_{\alpha}(x)^{s} \in C_{0}(\mathbb{R}), \quad m \rightarrow \infty
$$

$$
A:=\left\{f \in C(\mathbb{R}): f=\lim _{m \rightarrow \infty} w_{\alpha}^{m} g_{m}, g_{m} \in P_{m}\right\}
$$

then it follows that $x^{k}|x|^{\alpha} w_{\alpha}(x)^{s} \in A$ whenever $k \geq 0, s>p+k$. Just as above this means that $Z_{A}=\{0, \infty\}$ and we have suitable test functions in the closed subalgebra $A \subset C_{0}(\mathbb{R})$ which separate points in $\mathbb{R} \backslash\{0, \infty\}$. Hence, another application of the Stone-Weierstrass theorem implies that $A=C_{0}(\mathbb{R})$.

Remark. The construction in this section allows us to show that the estimate in Lemma 3 is sharp. Indeed, let

$$
M_{n}\left(w_{\alpha}\right):=\sup \left\{\left\|w_{\alpha}^{n} p_{n}^{\prime}\right\|_{\mathbb{R}}: p_{n} \in P_{n},\left\|w_{\alpha}^{n} p_{n}\right\|_{R} \leq 1\right\}
$$

be the $n$-th order weighted Markov factor. Lemma 3 claims that this is $\leq c_{1} n^{1 / \alpha}$. Now we show that if $0<\alpha<1$ is rational, then $M_{n}\left(w_{\alpha}\right) \geq c_{2} n^{1 / \alpha}, n=1,2, \ldots$, with some $c_{2}>0$.

Indeed, let $\alpha=p / q$, and for $n=4,5, \ldots$ set

$$
h_{p n}(x):=\frac{1}{2 q} \sum_{k=1}^{2 q}\left(1+t_{k}|x|^{\alpha}\right)^{q n}=\sum_{2 r \leq n} b_{r, n} x^{2 r p},
$$

where $t_{k}, 1 \leq k \leq 2 q$, are the $2 q$-th roots of unity and $b_{r, n}:=\left(\begin{array}{c}q n \\ 2 r q\end{array}\right)$. This formula and the fact that $h_{p n}$ is a polynomial of degree at most $p n$ can be verified similarly to $(9)$. Obviously, $w_{\alpha}^{p n}(x)\left|h_{p n}(x)\right| \leq 1$ on the whole real line, so we have

$$
b_{1, n}=\frac{h_{p n}^{(2 p)}(0)}{(2 p) !} \leq \frac{M_{p n}\left(w_{p / q}\right)^{2 p}}{(2 p) !},
$$

which, in view of $b_{1, n} \geq n^{2 q} /(2 q)$ !, yields

$$
M_{p n}\left(w_{\alpha}\right) \geq c_{2}^{\prime} n^{q / p}=c_{2}^{\prime} n^{1 / \alpha}
$$

and this implies $M_{n}\left(w_{\alpha}\right) \geq c_{2} n^{1 / \alpha}$.

For irrational $0<\alpha<1$ the just given argument implies that for every $\epsilon>0$ we have $M_{n}\left(w_{\alpha}\right) \geq c_{\epsilon} n^{\frac{1}{\alpha}-\epsilon}$. Indeed, let $\alpha<p / q<1$, where $p, q$ are positive integers, and consider the polynomial $h_{p n}$ from (13). If $\beta>1$, then the fraction $\left(1+t^{\beta}\right) /(1+t)^{\beta}$ is decreasing on $(0,1)$ and increasing on $(1, \infty)$, furthermore at $t=0$ and $t=\infty$ it is 1 , so it is always at most 1 . This implies ( $\operatorname{set} t=|x|^{\alpha}$ and $\beta=p / q \alpha$ ) that

$$
\frac{w_{\alpha}(x)}{w_{p / q}(x)}=\left(\frac{1+\left(|x|^{\alpha}\right)^{p / q \alpha}}{\left(1+|x|^{\alpha}\right)^{p / q \alpha}}\right)^{q / p} \leq 1
$$


for all $x$. Therefore, together with $w_{p / q}^{n}(x)\left|h_{p n}(x)\right| \leq 1$, we also have $w_{\alpha}^{n}(x)\left|h_{p n}(x)\right| \leq 1$. Now (14) yields as before

$$
M_{p n}\left(w_{\alpha}\right) \geq c_{2, p, q} n^{q / p}
$$

for all $n$, which implies

$$
M_{n}\left(w_{\alpha}\right) \geq c_{p, q} n^{q / p}
$$

Since here $q / p<1 / \alpha$ can be arbitrarily close to $1 / \alpha, M_{n}\left(w_{\alpha}\right) \geq c_{\epsilon} n^{\frac{1}{\alpha}-\epsilon}$ follows.

\section{References}

[1] D. Benko and A. Kroó, A Weierstrass-type theorem for homogeneous polynomials, Trans. Amer. Math. Soc., 361(2009), 1645-1665.

[2] R.P. Boas, Entire functions, Academic Press, New York, 1954.

[3] R. A. DeVore and G. G. Lorentz, Constructive approximation, Grundlehren der mathematischen Wissenschaften, 303, Springer Verlag, 1993.

[4] A. Kroó and J. Szabados, On the density of homogeneous polynomials on regular convex surfaces, Acta Sci. Math., 75(2009), 143-159.

[5] A. B. J. Kuijlaars, A note on weighted polynomial approximation with varying weights, J. Approx. Th., 87(1996), 112-115.

[6] T. Ransford, Potential theory in the complex plane, Cambridge University Press, Cambridge, 1995.

[7] E. B. Saff and V. Totik, Logarithmic potentials with external fields, Grundlehren der mathematischen Wissenschaften, 316, Springer Verlag, Berlin, 1997.

[8] V. Totik, Approximation by homogeneous polynomials, J. Approx. Th., 174 (2013), $192-205$.

[9] P. Varjú, Approximation by homogeneous polynomials, Constr. Approx., 26,(2007), 317-337.

\section{András Kroó}

Alfréd Rényi Institute of Mathematics

Hungarian Academy of Sciences

Budapest, Hungary

and

Budapest University of Technology and Economics

Department of Analysis, Budapest, Hungary

\section{Vilmos Totik}

Bolyai Institute, MTA-SZTE Analysis and Stochastics Research Group

University of Szeged, Szeged, Aradi v. tere 1, 6720, Hungary

and

Department of Mathematics and Statistics

University of South Florida

4202 E. Fowler Ave, CMC342, Tampa, FL 33620-5700, USA 